\title{
Reliability Evaluation of Uncertain Multi-State Systems based on Weighted Universal Generating Function
}

\author{
Wenjie Dong ${ }^{*}$, Sifeng Liu, Zhigeng Fang, and Yingsai Cao
}

College of Economics and Management, Nanjing University of Aeronautics and Astronautics, Jiangsu, 211106, China

\begin{abstract}
Universal generating function (UGF) is a basic and important technology in the reliability evaluation of multi-state systems (MSSs). It has been widely noticed by reliability scholars and engineers since its introduction. In the process of reliability evaluation of MSSs with UGF, universal generating operators play a great role in synthesizing the system output performance rate. For many uncertain MSSs in actual engineering, when the connection structure between components is unknown and/or the performance relationship is unclear, the definition of the weighted universal generating function is proposed. By designing reliability evaluation indices and constructing a weighted universal generating function of MSSs, reliability parameters of MSSs can be evaluated. A real case of steam turbine power generation system in a repairable naval equipment system is conducted to illustrate the applications.
\end{abstract}

Keywords: multi-state system; universal generating function; weight determination; reliability evaluation; steam turbine power generation system

(Submitted on October 21, 2018; Revised on November 17, 2018; Accepted on December 12, 2018)

(C) 2019 Totem Publisher, Inc. All rights reserved.

\section{Nomenclature}

$\begin{array}{ll}S & \text { A multi-state system } \\ j & \text { Multi-state component } \\ k_{j} & \text { Number of states of component } j \\ G_{j}(t) & \text { Performance rate of component } j \text { at time } t \\ P_{j}(t) & \text { Corresponding state possibility of component } j \text { at time } t \\ u_{j}(z, t) & \text { UGF of component } j \text { at time } t \\ u(z, t) & \text { UGF of } S \text { at time } t \\ f & \text { Universal generating operator } \\ f_{s e r} & \text { Universal generating operator of series systems } \\ f_{p a r} & \text { Universal generating operator of parallel systems } \\ K & \text { Number of states of } S \\ L^{n} & \text { Performance rate space of } S \\ S_{1} & \text { A flow transmission MSS } \\ S_{2} & \text { A task processing MSS } \\ T_{j} & \text { Task processing time of component } j \text { in } S_{2} \\ A(t, w) & \text { Availability } \\ \delta_{A} & \text { Availability operator } \\ E(t) & \text { Mean output performance }\end{array}$

* Corresponding author.

E-mail address: dongwenjie@nuaa.edu.cn 


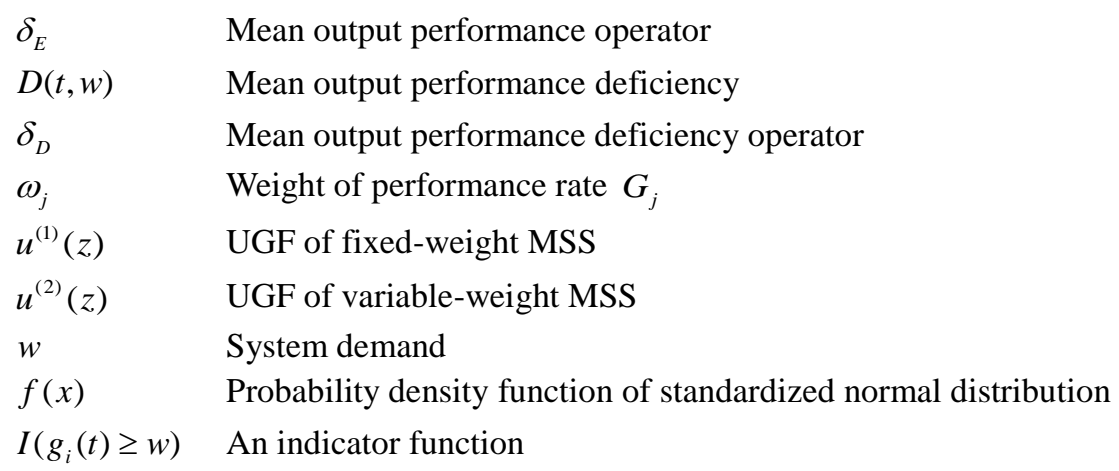

\section{Introduction}

In traditional reliability theory, we usually assume only two possible states for a system and its components: perfect functionality and complete failure. In other words, state 1 represents a system working well while 0 represents it failing. However, for most systems in real engineering, in addition to two states of perfect functionality and complete failure, there are also a series of deteriorating states [1-4]. For example, in a power generation system, when the generator generates a power of $100 \mathrm{MW}$, the system is in a normal state, and when it generates a power of $0 \mathrm{MW}$, the system is in a state of complete failure. However, due to some types of faults, the generated power may be $80 \mathrm{MW}$ at one time and $60 \mathrm{MW}$ at another time. These systems are called multi-state systems (MSSs). Actually, a binary system is the simplest multi-state system containing two distinctive states 1 and 0 .

Since the concept of MSSs was proposed, many scholars have spread their research on the reliability of MSSs [5-7]. From qualitative assessment to quantitative analysis, from the same number of states to different numbers, and from an ordered set to a partially ordered set of the description of state space, four main methods of reliability evaluation for MSSs have been gradually formed: an extension of the Boolean model [8], stochastic process method [9], universal generating function (UGF) technology [10-12], and Monte-Carlo simulation [13]. Due to the advantages of convenient computation and easy analysis, UGF has been widely studied in reliability evaluation and optimization of MSSs. It has gradually become one of the most basic and commonly used methods for the reliability assessment of MSSs [2, 14-15].

Based on the z-transform of discrete random variables, the state performance and corresponding possibility of multistate components can be represented by a $u$ function. According to the connection structure between components and performance characteristics, we can design universal generating operators [16] and then obtain polynomial representation of the whole system. Finally, we can evaluate and optimize the reliability of MSSs combined with system demand. In the process of reliability evaluation of MSSs, universal generating operators have a significant influence on output performance and state probability of the whole system, which will affect the reliability of MSSs to a great extent.

In the process of reliability management, when the connection relationship between components is unknown and the system performance is uncertain [17], assigning a proper weight to the multi-state component performance and constructing a more general UGF will be important for evaluating and optimizing the reliability of MSSs. Therefore, on the basis of UGF, this paper introduces the Ordered Weighted Averaging (OWA) [18] operator into weight determination when calculating system output performance. By designing reliability evaluation indices of MSSs and constructing a weighted universal generating function, reliability parameters of MSSs [19-20] can be evaluated. The case starts from a steam turbine power generation system, proving the practicability of the method proposed in this paper through a comparison with the traditional universal generating function.

The organization of this paper is as follows. Section 2 describes the basic synthetic methods of system performance according to connection relationships between components and performance characteristics and then proposes three main reliability indices in the reliability evaluation of MSSs. Section 3 puts forward the definition of weighted universal generating function and establishes two methods of weight determination of system performance. In Section 4 , for a steam turbine power generation system in a repairable naval equipment system where the connection structures between components are unknown, we use four styles of weighted universal generating function to evaluate the multi-state system's reliability. Finally, we present conclusions of this paper in Section 5. 


\section{Universal Generating Function}

\subsection{System Performance Associated with Connection Structures Between Components}

Suppose that an MSS $S$ has $n$ components and each component $j(j=1,2, \cdots, n)$ has $k_{j}$ states. The performance rate of $j$ at time $t$ is $G_{j}(t)$ and $G_{j}(t) \in\left\{g_{j 1}(t), g_{j 2}(t), \cdots, g_{j k_{j}}(t)\right\}$, the corresponding state probability is $P_{j}(t)$, and $P_{j}(t) \in\left\{p_{j 1}(t), p_{j 2}(t), \cdots, p_{j k_{j}}(t)\right\}$. Then, the universal generating function of multi-state component $j$ is

$$
u_{j}(z, t)=\sum_{i=1}^{k_{j}} p_{j i}(t) \cdot z^{g_{j i}(t)}
$$

The universal generating function of the multi-state system $S$ is

$$
u(z, t)=\sum_{j_{1}=1}^{k_{1}} \sum_{j_{2}=1}^{k_{2}} \cdots \sum_{j_{n}=1}^{k_{n}}\left(p_{1 j_{1}}(t) p_{2 j_{2}}(t) \cdots p_{n j_{n}}(t)\right) \cdot z^{f\left(g_{1 j_{1}}(t) g_{2 j_{2}}(t) \cdots g_{n_{n}}(t)\right)}
$$

In Equation (2), $f$ is the system structure function and is also called the universal generating operator. In general, $f_{\text {ser }}$ is used to represent the series connection while $f_{p a r}$ is used to represent the parallel connection. From Equation (2), we can see that the number of states of multi-state system $S$ is

$$
K=k_{1} k_{2} \cdots k_{n}=\prod_{j=1}^{n} k_{j}
$$

The performance rate space is $L^{n}=\left\{g_{11}, g_{12}, \cdots, g_{1 k_{1}}\right\} \times\left\{g_{21}, g_{22}, \cdots, g_{2 k_{2}}\right\} \times \cdots \times\left\{g_{n 1}, g_{n 2}, \cdots, g_{n k_{n}}\right\}$. Suppose that the performance capability of $j(j=1,2, \cdots, n)$ is $G_{j}$ and $G_{j} \in\left\{g_{j 1}, g_{j 2}, \cdots, g_{j k_{j}}\right\},(j=1,2, \cdots, n)$. There are two common types of MSS: flow transmission MSS and task processing MSS. For the series system $S_{1}$ constituted by multi-state components as shown in Figure 1(a), when $S_{1}$ is a flow transmission MSS and performance $G_{j}(j=1,2, \cdots, n)$ represents the transmission capability, the total transition capability of $S_{1}$ depends on the bottleneck component.

$$
G_{s}=f_{\text {ser }}^{(1)}=\min \left\{G_{1}, G_{2}, \cdots, G_{n}\right\}
$$

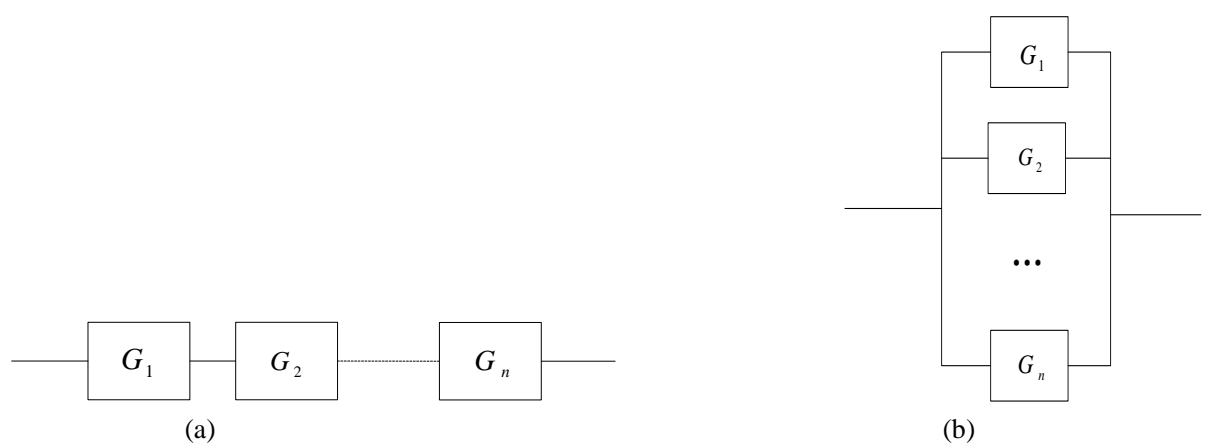

Figure 1. (a) A multi-state series system; (b) A multi-state parallel system

When $S_{1}$ is a task processing MSS and performance $G_{j},(j=1,2, \cdots, n)$ represents the processing speed, the task processing time of component $j$ is $T_{j}=1 / G_{j},\left(G_{j} \neq 0\right)$. The total performance rate is

$$
G_{s}=f_{s e r}^{(2)}=\frac{1}{T_{s}}=\frac{1}{\sum_{j=1}^{n} T_{j}}=\frac{1}{\sum_{j=1}^{n}\left(1 / G_{j}\right)}
$$


For the parallel system $S_{2}$ constituted by multi-state components as shown in Figure 1(b), when $S_{2}$ is a flow transmission MSS, the total performance rate of $S_{2}$ is

$$
G_{s}=f_{p a r}^{(1)}=\sum_{j=1}^{n} G_{j}
$$

When $S_{2}$ is a task processing MSS, the total performance rate of $S_{2}$ is

$$
G_{s}=f_{p a r}^{(2)}=\max \left\{G_{1}, G_{2}, \cdots, G_{n}\right\}
$$

Therefore, the total performance rate $G_{s}$ is decided by the connection relationship (Figure 1(a) and Figure 1(b)) and system performance characteristics, as shown in Table 1.

\begin{tabular}{c|c|c}
\multicolumn{2}{c}{ Table 1. Relationship between system performance and component performances } \\
\hline \multirow{4}{*}{ Series system } & Type of MSS & System performance \\
\cline { 2 - 3 } & Flow transmission MSS & $G_{s}^{(1)}=\min \left\{G_{1}, G_{2}, \cdots, G_{n}\right\}$ \\
\cline { 2 - 3 } & Task processing MSS & $G_{s}^{(2)}=\frac{1}{\sum_{j=1}^{n}\left(1 / G_{j}\right)}$ \\
\hline \multirow{3}{*}{ Parallel system } & Flow transmission MSS & $G_{s}^{(3)}=\sum_{j=1}^{n} G_{j}$ \\
\cline { 2 - 3 } & Task processing MSS & $G_{s}^{(4)}=\max \left\{G_{1}, G_{2}, \cdots, G_{n}\right\}$ \\
\hline
\end{tabular}

From Table 1, we can see that the total performance rate $G_{s}$ can be regarded as a certain function relationship of $G_{1}, G_{2}, \cdots, G_{n}$, that is, $G_{s}=f\left(G_{1}, G_{2}, \cdots, G_{n}\right) \cdot f(\cdot)$ is generally decided by the connection relationship between components and system performance characteristics. In the general series-parallel system shown in Figure 2, the relationship between $G_{s}$ and $G_{j}(j=1,2, \cdots, n)$ can be represented as

$$
G_{s}=f_{s e r}\left\{f_{p a r}\left(G_{11}, G_{12}, \cdots, G_{1 n}\right), \cdots, f_{p a r}\left(G_{m 1}, G_{m 2}, \cdots, G_{m n}\right)\right\}
$$

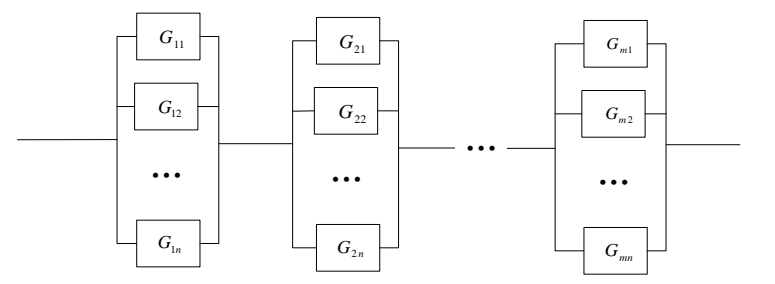

Figure 2. A general series-parallel system

\subsection{Reliability Evaluation Indices of MSSs}

System reliability refers to the ability of a product to complete the prescribed function under specified conditions and time, and we often use time function $R(t)$ to measure the reliability of a system or a product. Suppose in a constant condition profile, the demand of an MSS is $w$ at time $t$. The system performance is $G_{s}(t) \in\left\{g_{1}(t), g_{2}(t), \cdots, g_{K}(t)\right\}$ and the corresponding possibility is $P_{s}(t) \in\left\{p_{1}(t), p_{2}(t), \cdots, p_{K}(t)\right\}$, in which $K$ is the total number of system performances and according to Equation (3), $K=\prod_{j=1}^{n} k_{j}$. In general, the reliability measurement parameters include availability $A(t, w)$, mean output performance $E(t)$, and mean output performance deficiency $D(t, w)$.

Availability of an MSS $S$ is the possibility that the system performance satisfies the demand, namely, 


$$
A(t, w)=\delta_{A}(u(z, t), w)=\sum_{i=1}^{K} p_{i}(t) I\left(g_{i}(t) \geq w\right)
$$

In Equation (9), $\delta_{A}$ is called the availability operator, $I\left(g_{i}(t) \geq w\right)$ is an indicator function, and

$$
I\left(g_{i}(t) \geq w\right)= \begin{cases}1, & g_{i}(t) \geq w \\ 0, & g_{i}(t)<w\end{cases}
$$

Then, Equation (9) can be transferred to

$$
A(t, w)=\sum_{g_{i}(t) \geq w} p_{i}(t)
$$

When $t \rightarrow+\infty$, we can obtain the stable availability of an MSS

$$
A_{\infty}(w)=\sum_{g_{k} \geq w} p_{k}
$$

The mean output performance $E(t)$ of an MSS $S$ is the average value of the system output performance, namely,

$$
E(t)=\delta_{E}(u(z, t), w)=\sum_{i=1}^{K} g_{i}(t) p_{i}(t)
$$

In Equation (13), $\delta_{E}$ is called the mean output performance operator. From Equation (13), we can see that the mean output performance $E(t)$ of an MSS has nothing to do with the demand $w$.

When $t \rightarrow+\infty$, we can obtain the stable mean output performance of an MSS:

$$
E_{\infty}=\sum_{k=1}^{K} g_{k} p_{k}
$$

The mean output performance deficiency of an MSS is the average value when the system output performance does not satisfy demand, namely,

$$
D(t, w)=\delta_{D}(u(z, t), w)=\sum_{i=1}^{K} p_{i}(t) \max \left\{w-g_{i}(t), 0\right\}=\sum_{w \geq g_{i}(t)} p_{i}(t)\left(w-g_{i}(t)\right)
$$

In Equation (15), $\delta_{D}$ is called the mean output performance deficiency operator. When $t \rightarrow+\infty$, we can obtain the stable mean output performance deficiency value of an MSS $S$ :

$$
D_{\infty}(w)=\sum_{w \geq g_{k}} p_{k}\left(w-g_{k}\right)
$$

\section{Reliability Evaluation of Uncertain MSS}

\subsection{Weighted Universal Generating Function}

From Equations (2) and (3), we can see that the total state numbers of an MSS is $K$, the system performance $f\left(g_{1 j_{1}}(t), g_{2 j_{2}}(t), \cdots, g_{n j_{n}}(t)\right)$ is the function of each component performance, and we can use $g_{k}(k=1,2, \cdots, K)$ to replace it. When the connection structure between components is unknown or the component performance appears to have uncertain characteristics, the total system performance $G_{s} \in\left\{g_{1}, g_{2}, \cdots, g_{K}\right\}$ cannot be determined by Equations (4) to (8). Anyway, 
$G_{s}$ can be represented as the sum of the weighted performance of $G_{1}, G_{2}, \cdots, G_{n}$, that is,

$$
G_{s}=f\left(G_{1}, G_{2}, \cdots, G_{n}\right)=\omega_{1} G_{1}+\omega_{2} G_{2}+\cdots+\omega_{n} G_{n}=\sum_{i=1}^{n} \omega_{i} G_{i}
$$

Where $\sum_{i=1}^{n} \omega_{i}=1$. Then, the weighted universal generating function (WUGF) can be defined as follows:

In an MSS, the performance of multi-state component $j(j=1,2, \cdots, n)$ is $G_{j}$, and its universal generating function is shown in Equation (1). When the connection structure between components is unknown or the component performance appears to have uncertain characteristics, the weighted universal generating function (WUGF) of the whole system is

$$
u(z)=\sum_{s=1}^{K} p_{s} \cdot z^{G_{s}}=\sum_{j_{1}=1}^{k_{1}} \sum_{j_{2}=1}^{k_{2}} \cdots \sum_{j_{n}=1}^{k_{n}}\left(p_{1 j_{1}}(t) p_{2 j_{2}}(t) \cdots p_{n j_{n}}(t)\right) \cdot z^{\sum_{i=1}^{n} \omega_{i} G_{i}}
$$

\subsection{Determination of System Performance Weight}

In the process of system performance determination, different weights are applied to component performances, which reflects the relative importance of each component in the MSS and will be more objective. Generally speaking, the weight determination methods include subjective experience, primary and secondary index queuing classification, expert survey, and so on. Different weight determination methods have greater impact on the final results of system reliability. The weight determination method based on the Ordered Weighted Averaging (OWA) operator is the most commonly used method in multi-attribute decision making, and it is widely used in every field such as production and life.

\section{1) Fixed Weight Universal Generating Function}

In order to reflect the principle of fairness, a greater weight should be applied to components with greater performance rate in an uncertain MSS [21]. That is, the weight $\omega_{j}$ of component $j(j=1,2, \cdots, n)$ has relations with its performance rate $G_{j} \in\left\{g_{j 1}, g_{j 2}, \cdots, g_{j k_{j}}\right\}$ and $\omega_{j}$ changes with $G_{j}$, as shown in Equation (19).

$$
\omega_{j}=\frac{G_{j}}{\sum_{j=1}^{n} G_{j}}
$$

Here, the MSS composed of the fixed weight universal generating function is called fixed-weight MSS, namely,

$$
u^{(1)}(z)=\sum_{j_{1}=1}^{k_{1}} \sum_{j_{2}=1}^{k_{2}} \cdots \sum_{j_{n}=1}^{k_{n}}\left(p_{1 j_{1}}(t) p_{2 j_{2}}(t) \cdots p_{n j_{n}}(t)\right) \cdot z^{\left(\sum_{i=1}^{n} G_{i}^{2} / \sum_{i=1}^{n} G_{i}\right)}
$$

Then, we can evaluate and optimize the reliability of a fixed weight MSS according to Equations (9) to (16) on the basis of system demand $w$.

2) Variable Weight Universal Generating Function

In literature [22], Xu proposed an OWA operator weight determination method based on normal distribution, and literature [23] improved this method starting from decision data. On the basis of considering performance data of a multistate component, we will improve the OWA operator to determine the component performance weight by taking from the standardized normal distribution.

Suppose that an MSS contains $n$ components and the performance of component $j(j=1,2, \cdots, n)$ is $G_{j} \in\left\{g_{j 1}, g_{j 2}, \cdots, g_{j k_{j}}\right\}$. For performance vector $\vec{G}=\left(G_{1}, G_{2}, \cdots, G_{n}\right)$, its expectation and standard deviation [24] are respectively 


$$
\mu=\frac{1}{n} \sum_{j=1}^{n} G_{j}, \sigma=\frac{1}{n} \sum_{j=1}^{n}\left(G_{j}-\mu\right)^{2}
$$

Then, after standardization of $\vec{G}=\left(G_{1}, G_{2}, \cdots, G_{n}\right)$, we can obtain $\vec{G}^{(s)}=\left(G_{1}^{(s)}, G_{2}^{(s)}, \cdots, G_{n}^{(s)}\right)$, in which

$$
G_{j}^{(s)}=\frac{G_{j}-\mu}{\sigma}
$$

Suppose the probability density function of standardized normal distribution $N(0,1)$ is $f(x)=\frac{1}{\sqrt{2 \pi}} \exp \left(-\frac{x^{2}}{2}\right)$ and the function value of standardized performance $\vec{G}^{(s)}=\left(G_{1}^{(s)}, G_{2}^{(s)}, \cdots, G_{n}^{(s)}\right)$ is $f\left(G_{1}^{(s)}\right), f\left(G_{2}^{(s)}\right), \cdots, f\left(G_{n}^{(s)}\right)$. Then, the performance weight of component $j$ is

$$
\omega_{j}=\frac{f\left(G_{j}^{(s)}\right)}{\sum_{j=1}^{n} f\left(G_{j}^{(s)}\right)}
$$

The weighted universal generating function of the whole MSS can be transferred to

$$
u^{(2)}(z)=\sum_{j_{1}=1}^{k_{1}} \sum_{j_{2}=1}^{k_{2}} \cdots \sum_{j_{n}=1}^{k_{n}}\left(p_{1 j_{1}}(t) p_{2 j_{2}}(t) \cdots p_{n j_{n}}(t)\right) \cdot z^{\left(\sum_{i=1}^{n} G_{i} f\left(G_{i}^{(s)}\right) / \sum_{i=1}^{n} f\left(G_{i}^{(s)}\right)\right)}
$$

Here, the MSS composed of the variable weight universal generating function is called the variable-weight MSS, and then we can measure the reliability indices according to the system demand $w$.

\section{Case Study}

For the naval equipment system, its structure is rather complex, and the integration level is very high. It involves a large number of high-tech achievements and independent core technology, so it is of great significance to control operation costs and make maintenance policies for carrying out reliability management of the naval equipment system. As the main power source of repairable naval equipment systems, the steam turbine power generation system is mainly made up of boilers, steam turbines, and dynamos. Steam produced by the boilers drives the turbines to rotate through pipes and convert mechanical energy into electric power under the electromagnetic induction principle. As the heart of steam turbine power generation systems, this power generation device is mainly composed of a main power unit and an auxiliary power unit, as shown in Figure 3.
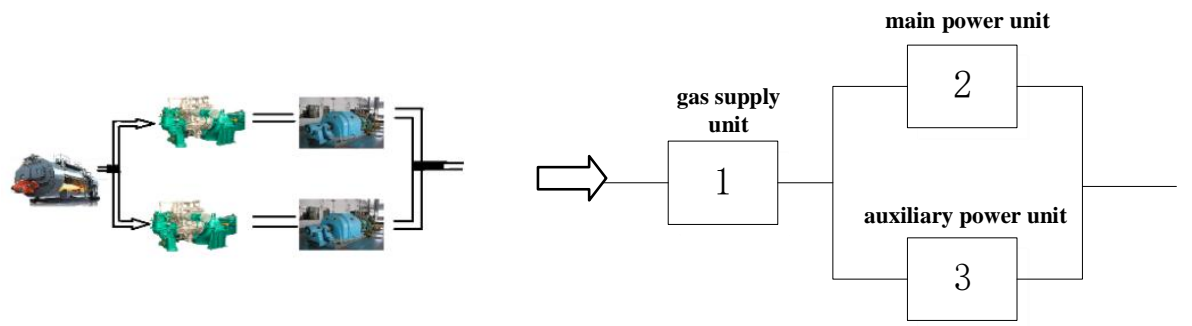

Figure 3. Structure of a steam turbine power generation system

The components of a steam turbine power generation system can be connected by the reliability block diagram shown in Figure 3. After the research on the inner characteristics of the boiler, steam turbine, and dynamo, we can divide them into multi-state components according to the percentage of normal operating capacity. For gas supply unit 1, the operating capacity is $90 \%$ of the designed power and it decreases to $60 \%$ during partial deterioration, so the performance vector of gas supply unit 1 is $G_{1} \in\left\{g_{11}=0, g_{12}=0.6, g_{13}=0.9\right\}$. For main power unit 2, the operating capacity can reach $100 \%$ of the designed power and it decreases to $60 \%$ during partial deterioration, so the performance vector of gas supply unit 1 is 
$G_{2} \in\left\{g_{21}=0, g_{22}=0.8, g_{23}=1.0\right\}$. The auxiliary power unit 3 is a typical binary-state system, that is, the performance vector is $G_{3} \in\left\{g_{31}=0, g_{32}=1.0\right\}$. Suppose the transfer between states of each component is a Markov process of continuous time. When the common cause failure phenomenon is not considered, the component performance parameters are shown in Table 2.

Table 2. Performance parameters of each component in the steam turbine power generation sys
\begin{tabular}{|c|c|c|c|}
\hline Component & Performance rate & Failure rate & Maintenance rate \\
\hline \multirow{3}{*}{1} & $g_{11}=0$ & - & $\mu_{12}^{(1)}=120$ \\
\cline { 2 - 4 } & $g_{12}=0.6$ & $\lambda_{21}^{(1)}=0.8$ & $\mu_{23}^{(1)}=150$ \\
\cline { 2 - 4 } & $g_{13}=0.9$ & $\lambda_{32}^{(1)}=1$ & - \\
\hline \multirow{3}{*}{2} & $g_{21}=0$ & - & $\mu_{12}^{(2)}=100$ \\
\cline { 2 - 4 } & $g_{22}=0.8$ & $\lambda_{21}^{(2)}=1.5$ & $\mu_{23}^{(2)}=120$ \\
\cline { 2 - 4 } & $g_{23}=1.0$ & $\lambda_{32}^{(2)}=2$ & - \\
\hline \multirow{2}{*}{3} & $g_{31}=0$ & - & $\mu_{12}^{(3)}=100$ \\
\hline & $g_{32}=1.0$ & $\lambda_{21}^{(3)}=0.3$ & - \\
\hline
\end{tabular}

Because the transition times between different states of each component are subject to exponential distribution, as shown in Figure 4, we can set up differential equations of the probability of every state of each component at time $t$ according to the Kolmogorov equation in the Markov process.

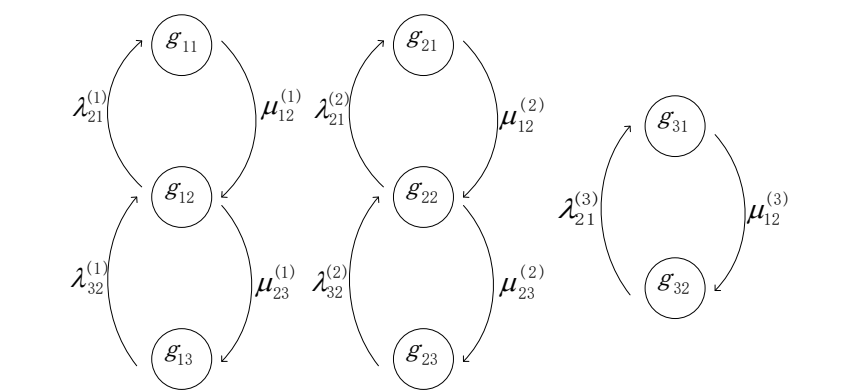

Figure 4. Transfer relationship between performance rate of each component

For components 1 and 2, namely when $i=1,2$,

$$
\left\{\begin{array}{l}
\frac{\mathrm{d} p_{i 1}(t)}{\mathrm{d} t}=\lambda_{21}^{(i)} p_{i 2}(t)-\mu_{12}^{(i)} p_{i 1}(t) \\
\frac{\mathrm{d} p_{i 2}(t)}{\mathrm{d} t}=\lambda_{32}^{(i)} p_{i 3}(t)-\left(\lambda_{21}^{(i)}+\mu_{23}^{(3)}\right) p_{i 2}(t)+\mu_{12}^{(3)} p_{i 1}(t) \\
\frac{\mathrm{d} p_{i 3}(t)}{\mathrm{d} t}=\mu_{23}^{(i)} p_{i 2}(t)-\lambda_{32}^{(i)} p_{i 3}(t) \\
p_{i 1}(t)+p_{i 2}(t)+p_{i 3}(t)=1
\end{array}\right.
$$

Suppose at the initial time $t=0, p_{i 1}(0)=0, p_{i 2}(0)=0$, and $p_{i 3}(0)=1$. After MATLAB programming, we can obtain $p_{i 1}(t), p_{i 2}(t)$, and $p_{i 3}(t)$, where $i=1,2$. For component 3 , because it is a typical binary system containing only two states, according to Kolmogorov equation:

$$
\left\{\begin{array}{l}
\frac{\mathrm{d} p_{31}(t)}{\mathrm{d} t}=\lambda_{21}^{(3)} p_{32}(t)-\mu_{12}^{(3)} p_{31}(t) \\
\frac{\mathrm{d} p_{32}(t)}{\mathrm{d} t}=\mu_{12}^{(3)} p_{31}(t)-\lambda_{21}^{(3)} p_{32}(t) \\
p_{31}(t)+p_{32}(t)=1
\end{array}\right.
$$


Suppose at $t=0, p_{31}(0)=0$ and $p_{32}(0)=1$, then we can also obtain $p_{31}(t)$ and $p_{32}(t)$ by means of MATLAB programming. According to Equation (1), the universal generating functions of each component are respectively.

$$
\begin{gathered}
u_{1}(z)=\sum_{j_{1}=1}^{3} p_{1 j_{1}}(t) \cdot z^{g_{1 j_{1}}}=p_{11}(t)+p_{12}(t) \cdot z^{0.6}+p_{13}(t) \cdot z^{0.9} \\
u_{2}(z)=\sum_{j_{2}=1}^{3} p_{2 j_{2}}(t) \cdot z^{g_{2 j_{2}}}=p_{21}(t)+p_{22}(t) \cdot z^{0.8}+p_{23}(t) \cdot z^{1} \\
u_{3}(z)=\sum_{j_{3}=1}^{2} p_{3 j_{3}}(t) \cdot z^{g_{3 j_{3}}}=p_{31}(t)+p_{32}(t) \cdot z^{1}
\end{gathered}
$$

According to Equation (2), the universal generating function of the steam turbine power generation system is

$$
u(z)=\sum_{j_{1}=1}^{3} \sum_{j_{2}=1}^{3} \sum_{j_{3}=1}^{2} p_{1 j_{1}}(t) p_{2 j_{2}}(t) p_{3 j_{3}}(t) \cdot z^{f\left(g_{1 j_{1}}, g_{2 j_{2}}, g_{3 j_{3}}\right)}
$$

From the system's universal generating function, we can determine that there are $3 \times 3 \times 2=18$ states in total. They are $f\left(g_{11}, g_{21}, g_{31}\right), \quad f\left(g_{11}, g_{21}, g_{32}\right), \quad f\left(g_{11}, g_{22}, g_{31}\right), \quad f\left(g_{11}, g_{22}, g_{32}\right), \quad f\left(g_{11}, g_{23}, g_{31}\right), \quad f\left(g_{11}, g_{23}, g_{32}\right), \quad f\left(g_{12}, g_{21}, g_{31}\right)$, $f\left(g_{12}, g_{21}, g_{32}\right), \quad f\left(g_{12}, g_{22}, g_{31}\right), \quad f\left(g_{12}, g_{22}, g_{32}\right), \quad f\left(g_{12}, g_{23}, g_{31}\right), \quad f\left(g_{12}, g_{23}, g_{32}\right), \quad f\left(g_{13}, g_{21}, g_{31}\right), \quad f\left(g_{13}, g_{21}, g_{32}\right)$, $f\left(g_{13}, g_{22}, g_{31}\right), f\left(g_{13}, g_{22}, g_{32}\right), f\left(g_{13}, g_{23}, g_{31}\right)$, and $f\left(g_{13}, g_{23}, g_{32}\right)$.

Let $G_{1} \in\left\{g_{11}, g_{12}, g_{13}\right\}, G_{2} \in\left\{g_{21}, g_{22}, g_{23}\right\}$, and $G_{3} \in\left\{g_{31}, g_{32}\right\}$, then the system performance can be represented as $G_{s}=f\left(G_{1}, G_{2}, G_{3}\right)$ uniformly. When the system demand $w=0.85$, we can obtain the change of availability, mean output performance, and output performance deficiency of the steam turbine power generation system according to Equations (9) to (16). As the connection structures of components are unknown, when synthesizing system performance, we can assume the system presents different performance characteristics and then calculate reliability indices according to Table 1 and the equations above. When the steam turbine power generation system is regarded as a flow transmission, a task processing MSS, a fixed-weight MSS, and a variable-weight MSS respectively, we find that reliability indices change with time, as shown in Figures 5 to 7.

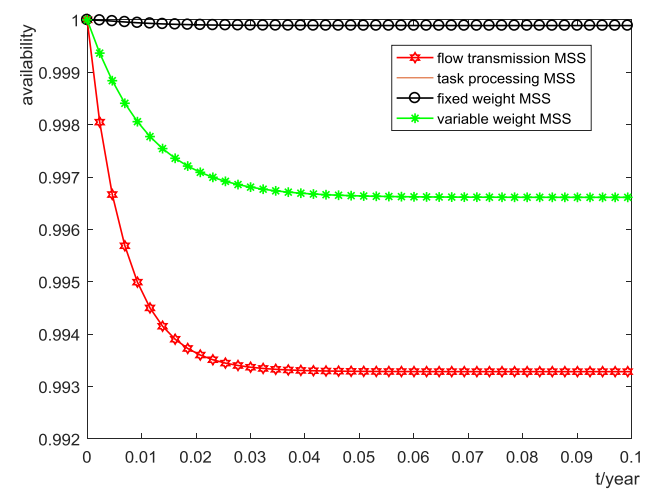

Figure 5. Availability of MSS

From Figure 5, we can see that the system availability $A(t, w)$ will reach a steady value at about $t=15$ days. When the performance relationships between components can be regarded as flow transmission, the stable availability of the whole system is 0.9933 , and when the relationships can be regarded as task processing, the stable availability is 0 . When the system performance is calculated by fixed weight UGF, as shown in 3.2, the stable availability is 0.9998; when the system performance is calculated by variable weight UGF, as shown in 3.2, the stable availability is 0.9966 . Obviously, only when the steam turbine power generation system is regarded as a task processing MSS will a large deviation appear in the system availability. When the system is regarded as a variable weight MSS, reliability indices are most consistent with the system characteristic itself, and then we can evaluate and optimize reliability parameters of the steam turbine power generation 
system. Similarly, for system mean output performance and mean performance deficiency, the stable values are shown in Table 3.

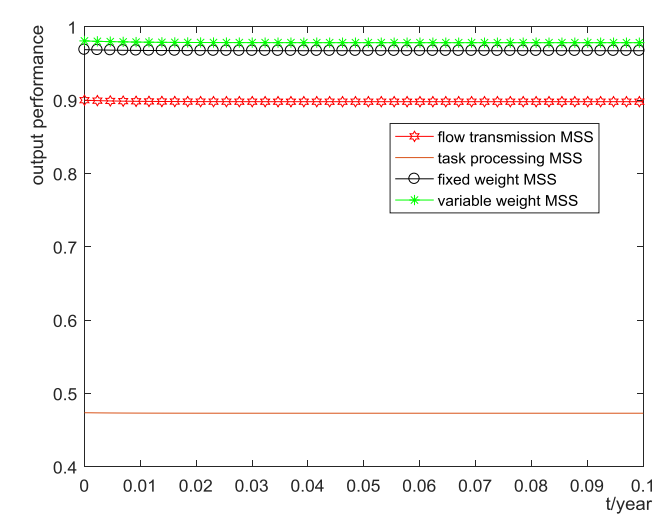

Figure 6. Mean output performance of MSS

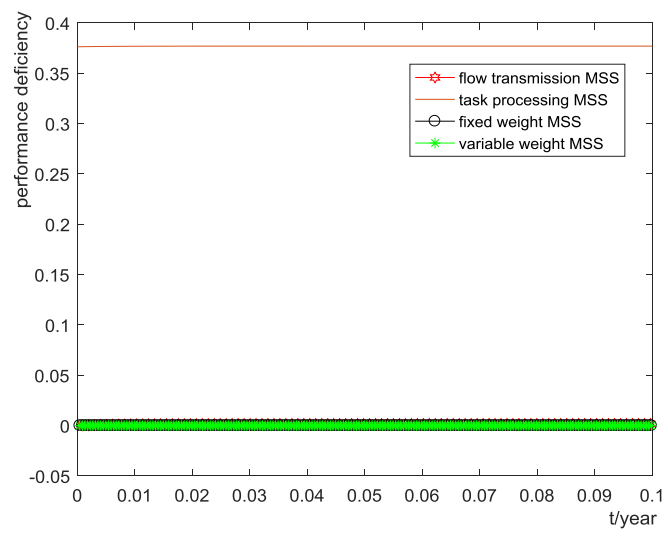

Figure 7. Mean performance deficiency of MSS

Table 3. Steady-state reliability indices values

\begin{tabular}{c|c|c|c}
\hline Rystem style & Stable availability & Stable output performance & Stable performance deficiency \\
\hline Flow transmission MSS & 0.9933 & 0.8980 & 0.0017 \\
\hline Task processing MSS & 0 & 0.4730 & 0.3770 \\
\hline Fixed weight MSS & 0.9998 & 0.9675 & $1.8489 \times 10^{-6}$ \\
\hline Variable weight MSS & 0.9966 & 0.9742 & $4.1884 \times 10^{-4}$ \\
\hline
\end{tabular}

From the above analysis, we can determine that when the connection relationship between components is unknown or the performance of the system is uncertain in an MSS, regardless of whether flow transmission or task processing is assumed, the system reliability will be unavoidably disturbed. The method of weighted UGF can deal with the reliability evaluation of uncertain MSSs to a certain extent. For this steam turbine power generation system, variable UGF can reach a better application effect, which is significant to the formulation of maintenance strategies.

\section{Conclusions}

The universal generating function is an important method for reliability evaluation and optimization of MSSs [25]. On the basis of UGF, aiming at uncertain systems or systems with unknown connection structures, this paper uses OWA operators to weight component performance, designs fixed weight UGF and variable weight UGF, and then synthesizes system output performance. After the comparison of output performance and system demand [26], three reliability evaluation parameters such as availability, mean output performance, and mean performance deficiency are defined. In the case study part, for an uncertain steam turbine power generation system with unknown component connection relationships, we first assume that its functional characteristics are flow transmission MSS and task processing MSS. By comparing and analyzing the proposed methods and existing methods, it is proven that the weighted universal generating function is scientific in the reliability evaluation of uncertain MSSs.

When synthesizing component performance, there are many ways to confirm weight [27]. On the basis of predecessors' research, this paper makes a reasonable empowerment of component performance through fixed weight and variable weight and obtains satisfactory results. In fact, when applying weighted UGF to the reliability evaluation of MSSs, the weight of each component's performance has a great influence on system reliability. Therefore, in engineering practice, the rational distribution of weight should be carried out according to the performance relationship between components in MSSs.

\section{Acknowledgements}

The authors would like to express their sincere appreciation for the reviewers for their useful suggestions to improve this paper. In addition, this work is supported by the National Natural Science Foundation of China (No. 71671091) and the China Postdoctoral Science Foundation (No. 2018M630561). 


\section{References}

1. I. B. Gertsbakh and M. Finkelstein, "Preventive Maintenance of Multistate Systems Subject to Shocks," Applied Stochastic Models in Business \& Industry, Vol. 32, No. 2, pp. 283-291, 2016

2. G. Levitin, "The Universal Generating Function in Reliability Analysis and Optimization," Springer London, 2005

3. A. Lisnianski, I. Frenkel, and Y. Ding, "Multi-State System Reliability Analysis and Optimization for Engineers and Industrial Managers," Springer London, 2010

4. K. S. Meena and T. Vasanthi, "Reliability Analysis of Mobile Ad Hoc Networks using Universal Generating Function," Quality \& Reliability Engineering International, Vol. 32, No. 1, pp. 111-122, 2016

5. R. E. Barlow and A. S. Wu, "Coherent Systems with Multi-State Components," Mathematics of Operations Research, Vol. 3, No. 4, pp. 275-281, 1978

6. G. Levitin, "A Universal Generating Function Approach for the Analysis of Multi-State Systems with Dependent Elements," Reliability Engineering \& System Safety, Vol. 84, No. 3, pp. 285-292, 2004

7. W. C. Yeh, "A New Approach to Evaluate Reliability of Multistate Networks under the Cost Constraint," Omega, Vol. 33, No. 3, pp. 203-209, 2005

8. A. Lisnianski, "Extended Block Diagram Method for a Multi-State System Reliability Assessment," Reliability Engineering \& System Safety, Vol. 92, No. 12, pp. 1601-1607, 2007

9. A. Lisnianski, "The Markov Reward Model for a Multi-State System Reliability Assessment with Variable Demand," Quality Technology \& Quantitative Management, Vol. 4, No. 2, pp. 265-278, 2007

10. Y. Ding, M. J. Zuo, and A. Lisnianski, "Fuzzy Multi-State Systems: General Definitions, and Performance Assessment," IEEE Transactions on Reliability, Vol. 57, No. 4, pp. 589-594, 2008

11. C. Y. Li, X. Chen, X. S. Yi, and J. Y. Tao, "Interval-Valued Reliability Analysis of Multi-State Systems," IEEE Transactions on Reliability, Vol. 60, No. 1, pp. 323-330, 2011

12. Y. F. Li, Y. Ding, and E. Zio, "Random Fuzzy Extension of the Universal Generating Function Approach for the Reliability Assessment of Multi-State Systems under Aleatory and Epistemic Uncertainties," IEEE Transactions on Reliability, Vol. 63, No. 1 , pp. 13-25, 2014

13. E. Zio, "The Monte Carlo Simulation Method for System Reliability and Risk Analysis," Springer Publishing Company, Incorporated, 2014

14. Y. F. Li and E. Zio, "A Multi-State Model for the Reliability Assessment of a Distributed Generation System via Universal Generating Function," Reliability Engineering \& System Safety, Vol. 106, No. 5, pp. 28-36, 2012

15. J. L. Qin, Y. G. Niu, and Z. Li, "A Combined Method for Reliability Analysis of Multi-State System of Minor-Repairable Components," Eksploatacja i Niezawodnosc - Maintenance and Reliability, Vol. 18, No. 1, pp. 80-88, 2016

16. P. Gao and L. Xie, "Reliability Analysis of Multi-State Systems based on Improved Universal Generating Function," Acta Aeronautica Et Astronautica Sinica, Vol. 31, No. 5, pp. 934-939, 2010

17. E. Zio, "Some Challenges and Opportunities in Reliability Engineering," IEEE Transactions on Reliability, Vol. 99, pp. 1-14, 2017

18. Yager and R. Ronald, "On Ordered Weighted Averaging Aggregation Operators in Multi-Criteria Decision Making," Readings in Fuzzy Sets for Intelligent Systems, Vol. 18, No. 1, pp. 80-87, 1993

19. G. Levitin, "Reliability of Multi-State Systems with Common Bus Performance Sharing," IIE Transactions, Vol. 43, No. 7, pp. 518-524, 2011

20. R. Peng, H. Xiao, and H. Liu, "Reliability of Multi-State Systems with a Performance Sharing Group of Limited Size," Reliability Engineering \& System Safety, Vol. 166, pp. 164-170, 2016

21. G. W. Wei and W. Yi, "A Method for Interval-Valued Intuitionistic Fuzzy Multiple Attribute Decision Making with Incomplete Weight Information," International Journal of Computational Intelligence Systems, No. 129, 2007

22. Z. S. Xu, "An Overview of Methods for Determining OWA Weights," International Journal of Intelligent Systems, Vol. 20, No. 8, pp. 843-865, 2005

23. Y. Wang and Z. S. Xu, "A New Method of Giving OWA Weights," Mathematics in Practice and Theory, Vol. 38, No. 3, pp. 51-61, 2008

24. Q. M. He, "Fundamentals of Matrix-Analytic Methods," Springer New York, 2014

25. C. Y. Li, X. Chen, and X. S. Yi, "Reliability Analysis of Primary Battery Packs based on the Universal Generating Function Method," Proceedings of the Institution of Mechanical Engineers Part O Journal of Risk \& Reliability, Vol. 223, No. 3, pp. 251-257, 2009

26. T. Nacef, E. Châtelet, and Y. Bouzidi, "How Combined Performance and Propagation of Failure Dependencies Affect the Reliability of a MSS," Reliability Engineering \& System Safety, Vol. 169, pp. 531-541, 2018

27. Y. Q. Luo, J. B. Xia, and T. P. Chen, "Comparison of Objective Weight Determination Methods in Network Performance Evaluation," Journal of Computer Applications, Vol. 29, No. 10, pp. 2624-2626, 2009

Wenjie Dong received his B.S. degree in industrial engineering from Shandong Jianzhu University, Jinan, China, in 2014, and he is currently working toward a Ph.D. in the College of Economics and Management at Nanjing University of Aeronautics and Astronautics, Nanjing, China. His research interests are reliability engineering and uncertainties.

Sifeng Liu is a professor in the College of Economics and Management at Nanjing University of Aeronautics and 
Astronautics, Nanjing, China. He received his Ph.D. in grey systems theory at Huazhong University of Science and Technology, Wuhan, China. He is the director of the Grey System Research Institute at Nanjing University of Aeronautics and Astronautics, the chairman of IEEE Grey System Society, and the vice chairman of IEEE SMC China (Beijing) Branch. His research interests include grey systems theory and complex equipment development and management. He is the editorin-chief of two international journals: Grey Systems: Theory and Application and Journal of Grey Systems.

Zhigeng Fang is a professor in the College of Economics and Management at Nanjing University of Aeronautics and Astronautics, Nanjing, China. His research fields are grey systems theory and reliability management.

Yingsai Cao received his B.S. degree in management science and engineering from Nanjing University of Aeronautics and Astronautics, China, in 2014, and he is currently working toward a Ph.D. in the College of Economics and Management at Nanjing University of Aeronautics and Astronautics. His research interests are reliability engineering and complex equipment development and management. 